\title{
Universality behaviour for polarity formation in channel-type inclusion compounds
}

\author{
Athanasios Batagiannis - Thomas Wüst • \\ Jürg Hulliger
}

Received: 14 March 2008 / Accepted: 26 June 2008 / Published online: 2 August 2008

(C) Springer Science+Business Media, LLC 2008

\begin{abstract}
A statistical investigation based on a Markov chain theory of polarity formation applied to channel-type inclusion compounds loaded with both dipolar $\mathrm{A}-\pi-\mathrm{D}$ and non-polar $\mathrm{N}-\pi-\mathrm{N}(\mathrm{N}$ : A or $\mathrm{D})$ guests is presented. The key parameters effecting polarity formation are identified and their effects are explored. A number of paradoxes are set out and an attempt to explain the mechanisms behind them is made: dependence of macroscopic polarity on orientational selectivity induced by intermolecular interactions, tuning of polarity through (i) the concentration of nonpolar guest and (ii) growth temperature.
\end{abstract}

Keywords Markov chain · Macroscopic polarity formation

\section{Introduction}

Experimental confirmation of basic features of growth induced polarity formation has been elaborated for various host systems suitable for inclusion of dipolar guest molecules into channels [1-4]. In view of a general theory of growth induced polarity formation [5], channel-type frames represent an ideal 1D system to apply a Markov chain description. By a Markov chain process here we understand a build-up process for the growth along the channel axis of inclusion compounds, where the "up" or "down" orientation of incoming dipolar guest molecules depends on probabilities which are determined by functional group interactions with previously included dipoles and the surface of the host material. In Fig. 1 basic assumptions are schematically summarized.

\footnotetext{
A. Batagiannis · T. Wüst · J. Hulliger $(\bowtie)$

Department of Chemistry and Biochemistry, University of Berne, Freiestrasse 3,

3012 Berne, Switzerland

e-mail: publication.hulliger@iac.unibe.ch
} 


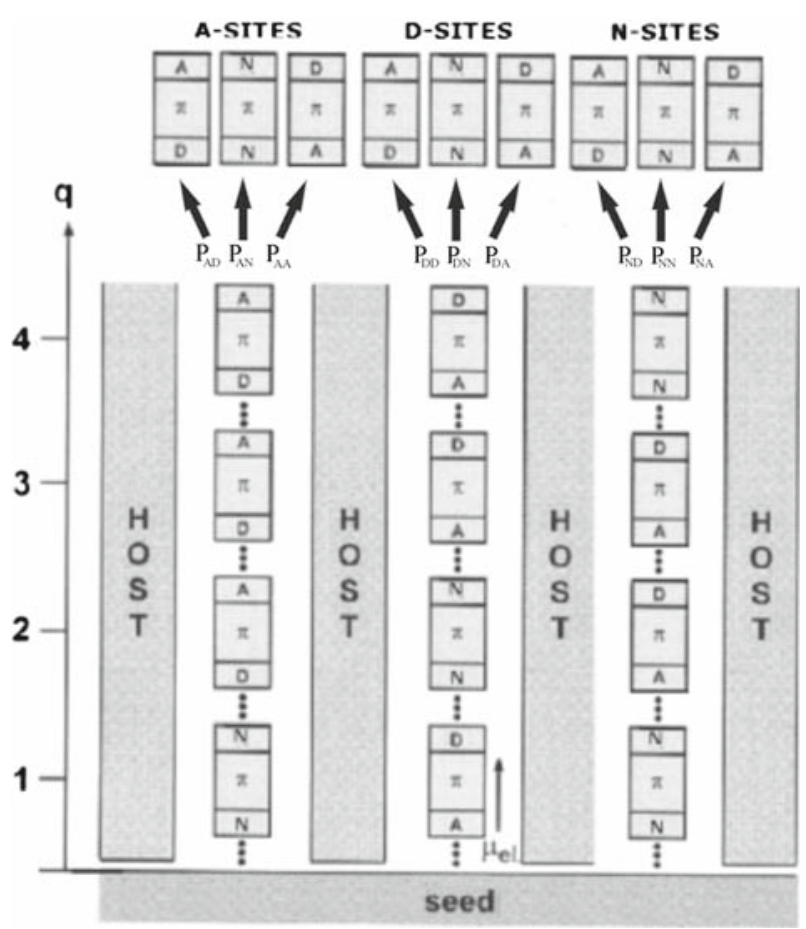

Fig. 1 Schematic representation of the host-guest lattice growing along the channel axis. Corresponding probabilities $P_{i j}$ are indicated. $q$ is the number of growth steps

A Markov chain model is suitable to describe this system because events for different channels are considered as independent. Furthermore, we consider a layerby-layer growth from the gas phase where one guest enters the channel at each time, interacting with guest molecules of the previously grown layer. Therefore, the memory of the system is restricted to the last layer on top of which the new layer is added. Such a $1 D$ series of events $(E)$ can be described by a simple probabilistic equation of the form:

$$
E(q+1)=P E(q)
$$

where $q=$ number of individual steps $(1,2, \ldots, \infty)$ and $P=$ transition probability from step $q$ to $q+1$, etc. This process is known as a first order homogeneous Markov chain and it is characterised by a development not dependent on initial conditions but only on the previous system state.

Here, we extend our theoretical analysis demonstrating a universality behaviour for channel-type hosts filled by a mixture of dipolar $\mathrm{A}-\pi-\mathrm{D}$ (A: acceptor, D: donor constituents of a $\pi$-conjugated bridging unit) and non-polar $\mathrm{N}-\pi-\mathrm{N}(\mathrm{N}$ : A or D) guest molecules. Because of specific $-\mathrm{A} \cdots \mathrm{N}-$ and $-\mathrm{D} \cdots \mathrm{N}$ - interactions new phenomena occur in such systems. It is found that the net fraction of aligned $\mathrm{A}-\pi-\mathrm{D}$ molecules $X_{\text {net }}=X_{\mathrm{A}}-X_{\mathrm{D}}$ (fractions of down and up oriented molecules) is a linear function 
of $X_{\mathrm{g}}$ and $X_{\mathrm{N}}$, the fractions of non-polar molecules present in the gas and those included in the host, respectively. Consequently, a compositional analysis for $X_{\mathrm{N}}$ allows to determine $X_{\text {net }}$, a quantity directly related to macroscopic polarity which can otherwise be obtained from e.g. scanning pyroelectric microscopy [6], second harmonic generation [7] or x-ray diffraction [8]. Surprisingly, calculations show that a maximum polar alignment can be achieved by admitting non-polar guest molecules. Therefore, the effect of dilution through a second guest is over-compensated by a non-linear enhancement of polarity.

\section{Theoretical model}

Taking advantage of fundamental knowledge on growth induced polarity formation [9-11] we can assume a time-homogeneous Markov chain description [12] being particularly valid in the case of lateral guest-guest interactions in the order of a small perturbation [13]. In case lateral interactions between channels become more significant, probabilities describing the step-by-step growth of polarity $\left(X_{\text {net }}\right)$ become themselves dependent on the local level of polarity as growth goes on. Here, we solve the problem using a single stochastic probability matrix $n \times n$. We set $n=3$ because we have the probabilities of inclusion of the "up" or "down" orientation of A- $\pi-\mathrm{D}$ guests and $\mathrm{N}-\pi-\mathrm{N}$ guests:

$$
\left(\begin{array}{c}
X_{\mathrm{A}} \\
X_{\mathrm{D}} \\
X_{\mathrm{N}}
\end{array}\right)=\left(\begin{array}{lll}
P_{\mathrm{AD}} & P_{\mathrm{DD}} & P_{\mathrm{ND}} \\
P_{\mathrm{AA}} & P_{\mathrm{DA}} & P_{\mathrm{NA}} \\
P_{\mathrm{AN}} & P_{\mathrm{DN}} & P_{\mathrm{NN}}
\end{array}\right)^{q}\left(\begin{array}{c}
X_{\mathrm{A}}^{0} \\
X_{\mathrm{D}}^{0} \\
X_{\mathrm{N}}^{0}
\end{array}\right)
$$

Making use of the general properties of transition probability matrices [12] for $q \rightarrow \infty$ (infinite number of growth layers), the following equations for $X_{\mathrm{A}}, X_{\mathrm{D}}$ and $X_{\mathrm{N}}$ were obtained for the stationary state:

$$
X_{\mathrm{A}}=\frac{P_{\mathrm{ND}}+P_{\mathrm{DD}} P_{\mathrm{NA}}-P_{\mathrm{DA}} P_{\mathrm{ND}}}{P_{\mathrm{ND}} P_{\mathrm{AA}}-P_{\mathrm{DD}} P_{\mathrm{AA}}+P_{\mathrm{DD}} P_{\mathrm{NA}}+P_{\mathrm{DA}} P_{\mathrm{AD}}-P_{\mathrm{DA}} P_{\mathrm{ND}}-P_{\mathrm{DA}}-P_{\mathrm{NA}} P_{\mathrm{AD}}+P_{\mathrm{NA}}-P_{\mathrm{AD}}+P_{\mathrm{ND}}+1}
$$

$$
=\frac{-P_{\mathrm{DA}} P_{\mathrm{AD}}+P_{\mathrm{NA}}+P_{\mathrm{ND}} P_{\mathrm{AA}}}{P_{\mathrm{ND}} P_{\mathrm{AA}}-P_{\mathrm{DD}} P_{\mathrm{AA}}+P_{\mathrm{DD}} P_{\mathrm{NA}}+P_{\mathrm{DA}} P_{\mathrm{AD}}-P_{\mathrm{DA}} P_{\mathrm{ND}}-P_{\mathrm{DA}}-P_{\mathrm{NA}} P_{\mathrm{AD}}+P_{\mathrm{NA}}-P_{\mathrm{AD}}+P_{\mathrm{ND}}+1}
$$

$$
\begin{aligned}
& X_{\mathrm{N}} \\
& =\frac{-P_{\mathrm{DD}} P_{\mathrm{AA}}+P_{\mathrm{DA}} P_{\mathrm{AD}}-P_{\mathrm{DA}}-P_{\mathrm{AD}}+1}{P_{\mathrm{ND}} P_{\mathrm{AA}}-P_{\mathrm{DD}} P_{\mathrm{AA}}+P_{\mathrm{DD}} P_{\mathrm{NA}}+P_{\mathrm{DA}} P_{\mathrm{AD}}-P_{D A} P_{\mathrm{ND}}-P_{\mathrm{DA}}-P_{\mathrm{NA}} P_{\mathrm{AD}}+P_{\mathrm{NA}}-P_{\mathrm{AD}}+P_{\mathrm{ND}}+1}
\end{aligned}
$$

The probabilities of all possible interactions, $P_{i j}$, appearing in the matrix are functions of (i) the basic 1D guest-guest and host-guest interaction energies, (ii) the composition $X_{\mathrm{g}}$ (molar fraction of non-polar guest molecules $\mathrm{N}-\pi-\mathrm{N}$ in the gas 
Table 1 Guest-guest and host-guest interaction energies

\begin{tabular}{lc}
\hline$E_{i j}$ & Range $\left(\mathrm{kJ} \mathrm{mol}^{-1}\right)$ \\
\hline$E_{\mathrm{AD}}$ & -25.0 to 0.01 \\
$E_{\mathrm{AA}}$ & $0.01-10.0$ \\
$E_{\mathrm{DD}}$ & -5.0 to -5.0 \\
$E_{\mathrm{Cj}}$ & -5.0 to -5.0 \\
\hline
\end{tabular}

phase) and (iii) temperature. The probabilities $P_{i j}$ represent all possible attachments and are given by a Boltzmann factor multiplied by $X$, the availability of molecules in the nutrient, divided by the partition sum:

$$
P_{i j}=\frac{X e^{-\beta\left(E_{i j}+E_{C j}\right)}}{Z}
$$

where

$$
Z_{i}=\sum_{j \in\{\mathrm{A}, \mathrm{D}, \mathrm{N}\}} e^{-\beta\left(E_{i j}+E_{C j}\right)} ; \quad i \in\{\mathrm{A}, \mathrm{D}, \mathrm{N}\}
$$

$X$ being $X_{\mathrm{g}}$ for non-polar guests or $\left(1-X_{\mathrm{g}}\right)$ for polar guests. $\beta=1 / k_{\mathrm{B}} T . E_{C j}$ and $E_{i j}$ are the host-guest and guest-guest 1D interaction energies randomly chosen from the following ranges of energy given in Table 1.

Here it is assumed that $E_{\mathrm{NA}}=E_{\mathrm{NN}}=E_{\mathrm{AA}}$ and $E_{\mathrm{ND}}=E_{\mathrm{AD}}$ for $\mathrm{A}-\pi-\mathrm{A}$ guests and $E_{\mathrm{ND}}=E_{\mathrm{NN}}=E_{\mathrm{DD}}$ and $E_{\mathrm{NA}}=E_{\mathrm{AD}}$ for D- $\pi-\mathrm{D}$ guests.

These are probabilities related to individual interactions: Along the channel we have $\mathrm{A}-\pi-\mathrm{D} \cdots \mathrm{A}-\pi-\mathrm{D}, \mathrm{A}-\pi-\mathrm{D} \cdots \mathrm{D}-\pi-\mathrm{A}, \mathrm{D}-\pi-\mathrm{A} \cdots \mathrm{A}-\pi-\mathrm{D}$ and correspondingly $\mathrm{A}-\pi-\mathrm{D} \cdots \mathrm{N}-\pi-\mathrm{N}$ and $\mathrm{D}-\pi-\mathrm{A} \cdots \mathrm{N}-\pi-\mathrm{N}$ interactions. When entering a preexisting channel or part of it, the $\mathrm{A}-\pi-\mathrm{D}$ molecule may experience a significant energy difference depending on whether the A- or D-group enters first (see Fig. 1). Therefore, we introduce the energy terms $E_{\mathrm{CA}}$ and $E_{\mathrm{CD}}$ accounting for guest to channel wall interactions. In the case of non-polar molecules no such distinction is necessary. A detailed force field study on e.g. the inclusion of $p$-nitroaniline in tris $(o$-phenylenedioxy)cyclotriphosphazene (TPP) channels has revealed a significant energy difference, $\Delta E_{\mathrm{C}} \equiv E_{\mathrm{CA}}-E_{\mathrm{CD}}$ for entering an empty channel with the A- or D-group first [14]. Therefore, the present study will rather address channel forming host molecules such as perhydrotriphenylene (PHTP), where the effects of $\Delta E_{\mathrm{C}}$ are likely to be less pronounced than for TPP.

Concerning the guest-guest interactions we assume at first that corresponding $-\mathrm{A} \cdots \mathrm{D}-,-\mathrm{A} \cdots \mathrm{A}-$ and $-\mathrm{D} \cdots \mathrm{D}-$ for $\mathrm{A}-\pi-\mathrm{D}$ and $\mathrm{N}-\pi-\mathrm{N}$ molecules are the same or at least of similar order. Having discussed the system within this frame of an approximation including no inter-channel interactions, in the end we will also give an account on the general case. 


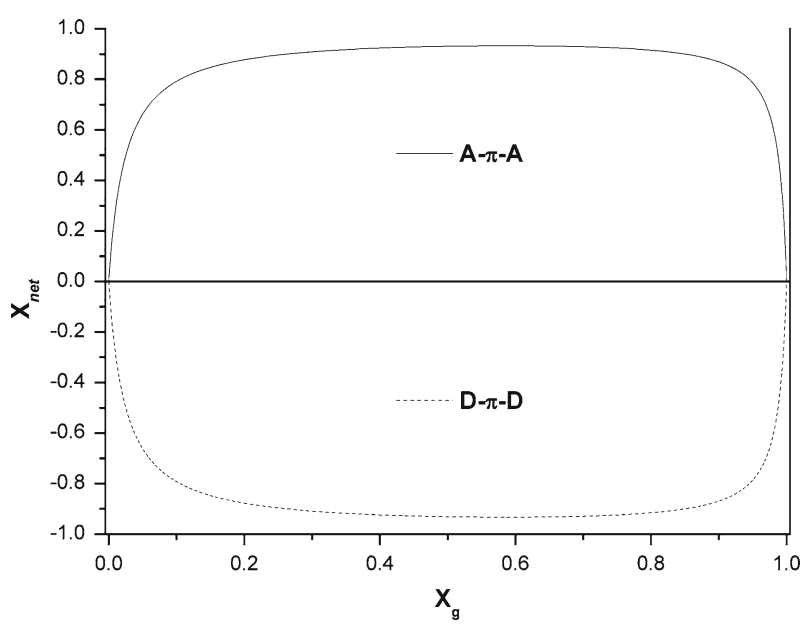

Fig. 2 The effects of adding $\mathrm{A}-\pi-\mathrm{A}$ (solid curve) or $\mathrm{D}-\pi-\mathrm{D}$ (dashed curve) guest molecules in the gas phase. The interaction energies were set to $E_{\mathrm{AD}}=-15 \mathrm{~kJ} \mathrm{~mol}^{-1}, E_{\mathrm{AA}}=E_{\mathrm{DD}}=1 \mathrm{~kJ} \mathrm{~mol}^{-1}$ and therefore $\Delta E_{\mathrm{f}}=0$. In this case polarity formation is only due to non-polar additives

\section{Results}

\subsection{Reviewing basic features}

Based on knowledge gained from previous studies [15] we can say that inclusion of $\mathrm{A}-\pi-\mathrm{D}$ molecules featuring positive values for $\Delta E_{\mathrm{A}} \equiv E_{\mathrm{AA}}-E_{\mathrm{AD}}$ and $\Delta E_{\mathrm{D}} \equiv$ $E_{\mathrm{DD}}-E_{\mathrm{AD}}$, will cause the system to develop into a bipolar growth state where A-groups appear preferably at the growing interface $(q \rightarrow \infty$, for ranges of possible interaction energies see Table 1$)$. Here, addition of $\mathrm{A}-\pi-\mathrm{A}$ to the growing system can enhance polarity formation $[11,16]$. Starting from a seed providing random orientations of $\mathrm{A}-\pi-\mathrm{D}$ in channels, preference for A-groups appearing at the surface is promoted.

A different situation is observed for $\mathrm{D}-\pi-\mathrm{D}$ co-guest molecules: Their addition can have a detrimental effect. For a positive $\Delta E_{\mathrm{f}} \equiv \Delta E_{\mathrm{A}}-\Delta E_{\mathrm{D}}$ as typically is the case for most common molecules, net polarity drops to zero, is inverted and a maximum value is obtained which is lower than that obtained when admitting no non-polar molecules $\left(X_{\mathrm{g}}=0\right)$. Experimental proof for this effect has been reported before [16].

Summarizing, a system characterized by $\Delta E_{\mathrm{f}}, \Delta E_{\mathrm{A}}$ and $\Delta E_{\mathrm{D}}$ all larger than zero (typical case), is influenced positively by the admixture of $\mathrm{A}-\pi-\mathrm{A}$ as long as it is not added in concentrations $X_{\mathrm{g}} \gtrsim 0.9$, whereas $\mathrm{D}-\pi-\mathrm{D}$ co-guest molecules appear detrimental. If, however, the interaction energies do not favour negative or positive polarity, i.e. $\Delta E_{\mathrm{A}}=0$, the effects of $\mathrm{A}-\pi-\mathrm{A}$ and $\mathrm{D}-\pi-\mathrm{D}$ are symmetrical and result in an enhancement of net polarity. Figure 2 illustrates the impact of non-polar guests on polarity: For both $\mathrm{A}-\pi-\mathrm{A}$ and $\mathrm{D}-\pi-\mathrm{D}$ guests we observe that once a certain critical amount of $X_{g}$ is co-included, polarity is maximised within the limit of dilution. A value of only $X_{g} \approx 0.2$ is sufficient to drive the system to an almost polar alignment. 


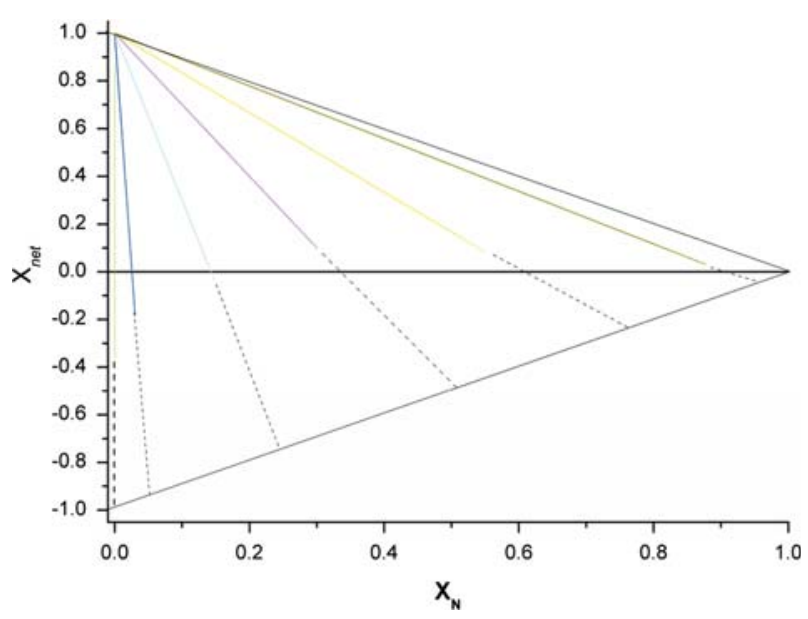

Fig. 3 Net polarity $X_{\text {net }}$, as a function of $X_{\mathrm{N}}$, the fraction of non-polar guests included in the crystal. $X_{\text {net }}$ is plotted for various values of $X_{\mathrm{g}}\left(-X_{\mathrm{g}}=0.05,-X_{\mathrm{g}}=0.25,-X_{\mathrm{g}}=0.5,-X_{\mathrm{g}}=0.75,-X_{\mathrm{g}}=0.95\right)$. For colour, see electronic version. Interactions energies were randomly chosen within the energy range given in Appendix 1. Dashed curves were obtained from calculations using an extended hypothetical energy range $\left(-100<E_{i j}<100 \mathrm{~kJ} \mathrm{~mol}^{-1}\right)$. The solid black lines show the upper limits of $X_{\text {net }}$ as a function of $X_{\mathrm{N}}$

This is an effect found also in solid solutions of molecular crystals and its impact on polarity is determined by the strength of the $-\mathrm{A} \cdots \mathrm{D}$ - interaction [17].

As shown before [9-11], macroscopic polarity in channel-type hosts filled with a single type of dipolar guests arises as a result of orientational selectivity driven by the energy difference between $-\mathrm{A} \cdots \mathrm{A}-$ and $-\mathrm{D} \cdots \mathrm{D}$ - interactions. For the set of interaction energies used for Fig. 2, $\Delta E_{\mathrm{f}}=0$ and hence polarity would not arise in the one component system. Once a non-polar guest is added, however, remarkably high values of $X_{\text {net }}$ can be achieved. This is a result of faulted orientations caused by the inclusion of non-polar guests and represents a paradox to our intuition.

\subsection{Universality behaviour}

Given the conservation equation $X_{\mathrm{A}}+X_{\mathrm{D}}+X_{\mathrm{N}}=1$, net polarity $X_{\text {net }}$ can only occur within a triangle limited by \pm 1 for $X_{\text {net }}$ and 1 for $X_{\mathrm{N}}$ (see Fig. 3). The linear curves appearing within the triangle result from a selected $X_{\mathrm{g}}$ (composition of growth) and $X_{\mathrm{N}}$ obtained by co-inclusion of $\mathrm{N}-\pi-\mathrm{N}$ molecules.

Analytical calculations show that the gradients $m$ of these solutions for polarity formation are simply given by (for details see Appendix 1):

$$
m=1-\frac{2}{X_{\mathrm{g}}}
$$

From this we obtain:

$$
X_{\text {net }}=1-X_{\mathrm{N}}\left(1-\frac{2}{X_{\mathrm{g}}}\right)
$$




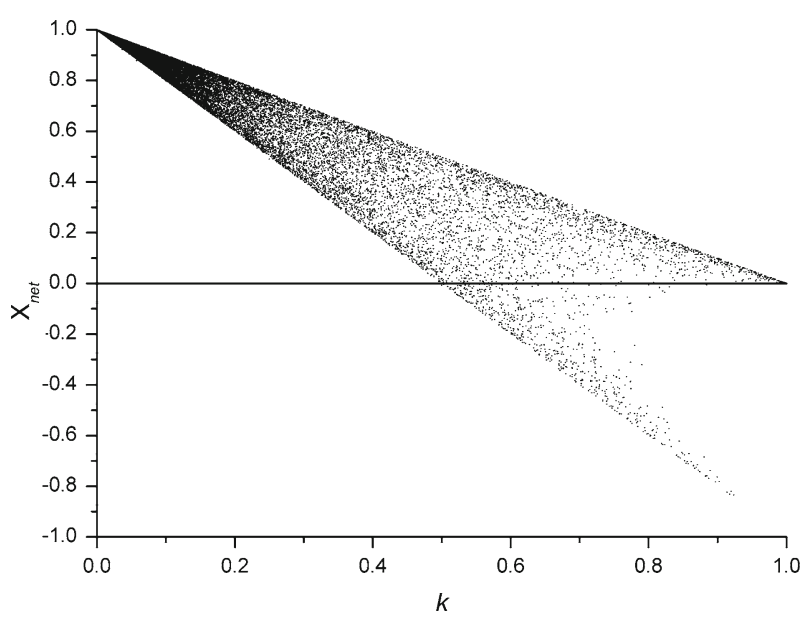

Fig. $4 X_{\text {net }}$ plotted as a function of the absorptivity $k=X_{\mathrm{N}} / X_{\mathrm{g}}$ of non-polar A- $\pi-\mathrm{A}$ guests. Interaction energies were randomly chosen from the fore mentioned energy set. The initial concentration of non-polar guests in the gas phase was randomly chosen from the range $0<X_{\mathrm{g}}<1 . T=300 \mathrm{~K}$. Lower values of $X_{\text {net }}$ are accessible for extended energy ranges

The signs in Eq. 8 are reversed for $\mathrm{D}-\pi-\mathrm{D}$ guests molecules. Equation 9 is independent of the interaction energies $E_{\mathrm{ij}}$ provided that the assumptions made above hold. Therefore, a compositional analysis allows $X_{\text {net }}$ to be determined without performing measurements involving physical properties (pyroelectricity [18] or second harmonic generation [18]). A small measured value of $X_{\mathrm{N}}(\mathrm{N}=\mathrm{A})$ would thus encourage us to conclude that $X_{\text {net }}$ may be close to one, i.e. almost full alignment is achieved. This is also valid in the case of $\mathrm{D}-\pi-\mathrm{D}$ guests inclusion, however, the sign of $X_{\text {net }}$ is reversed.

Equation 9 also shows the relationship between polarity and the uptake of neutral guest molecules, $k=X_{\mathrm{N}} / X_{\mathrm{g}}$. For $k=0$ the second term becomes zero and thus polarity is maximised. Increasing the amount of $\mathrm{N}-\pi-\mathrm{N}$ molecules means that less polar guests are included into the crystal and therefore maximum polarity has to be lower. For values of $k>0.5$ non-polar guests can also reverse the sign of $X_{\text {net }}$. When almost all of the non-polar molecules are co-included their effect on $X_{\text {net }}$ depends on their initial concentration, $X_{g}$ : for a small concentration polarity is strongly enhanced, whereas for higher concentrations polarity gradually diminishes due to dilution.

Figure 4 demonstrates the relationship between the amount of co-included $\mathrm{N}-\pi-\mathrm{N}$ molecules and $X_{\text {net }}$. The data plotted represent the results of 100,000 individual calculations performed using interaction energies randomly chosen from the energy ranges given in Table $1 . X_{\mathrm{g}}$ was also randomly chosen from values between 0 and 1 . The data clearly show that for a low value of $X_{\mathrm{N}}$ polarity is maximized within the limit of dilution. The mean value averaged over all points is $X_{\text {net }} \approx 0.91$ with a standard deviation $\sigma \approx 0.19$. Assuming a standard distribution, this corresponds to $\sim 68 \%$ of all points lying in the range denoted by the standard deviation. The high density of this distribution is illustrated on the top left corner of the graph in Fig. 4. Likewise, the data show that the uptake of $\mathrm{N}-\pi-\mathrm{N}$ molecules is generally low for $\mathrm{A}-\pi-\mathrm{A}$ guests. This is in accordance with experimental observations confirming that single inclusion 


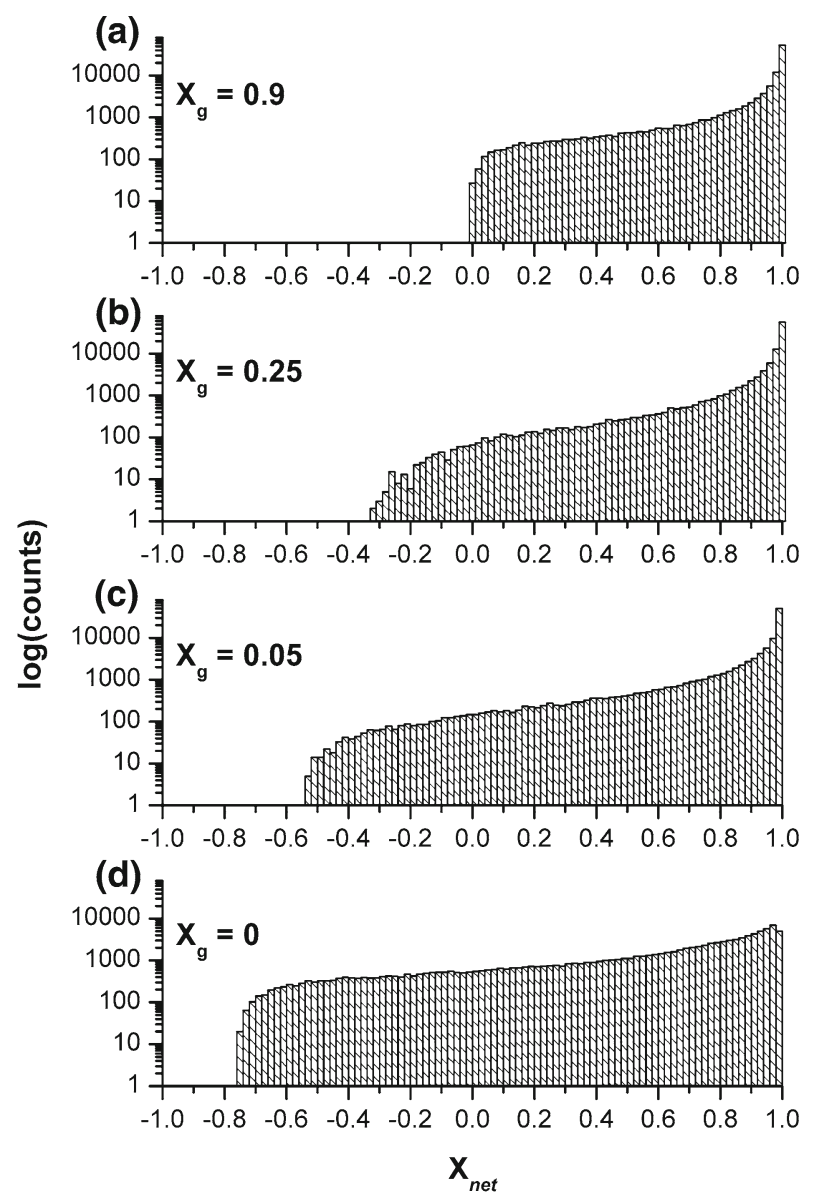

Fig. 5 Histograms of $X_{\text {net }}$ values obtained from 100,000 calculations for various values of $X_{\mathrm{g}}$ (a-d). Interaction energies are randomly chosen and the temperature was set to $300 \mathrm{~K}$ as before

of $\mathrm{A}-\pi-\mathrm{A}$ non-polar guests is difficult in most cases. The mean uptake is $k \approx 0.06$ with a standard deviation $\sigma \approx 0.14$.

In order to explore the effect of $\mathrm{A}-\pi-\mathrm{A}$ inclusion further, we have performed four sets of calculations for selected values of $X_{\mathrm{g}}$. The results are plotted on the histograms shown in Fig. 5. These histograms allow us to investigate where for a given range of interaction energies (same selection as before), the most probable $X_{\text {net }}$ may be found. The data were divided in bins of $\Delta X_{\text {net }}=0.02$ and the number of results within this range were counted for each bin. The higher the number of counts, the higher the probability that $X_{\text {net }}$ will be within this range regardless of the interaction energies chosen. We observe that even in the one component system $\left(X_{\mathrm{g}}=0\right)$ high polarity values have the highest probabilities (d). This tendency, however, is enhanced extremely (c) when non-polar guests in concentrations as little as $X_{\mathrm{g}}=0.05$ are added in the gas phase. This results from the increased probability of $-\mathrm{A} \cdots \mathrm{A}-$ interactions 
Table 2 Statistical measures for histograms shown in Fig. 4

\begin{tabular}{llll}
\hline$X_{\mathrm{g}}$ & Mean of $X_{\text {net }}$ & $\sigma^{\mathrm{a}}$ & $\begin{array}{l}\text { Range of } \\
X_{\text {net }}(\max =2)\end{array}$ \\
\hline$X_{\mathrm{g}}=0$ & 0.57 & 0.42 & 1.75 \\
$X_{\mathrm{g}}=0.05$ & 0.87 & 0.24 & 1.54 \\
$X_{\mathrm{g}}=0.25$ & 0.92 & 0.17 & 1.32 \\
$X_{\mathrm{g}}=0.9$ & 0.90 & 0.19 & 1.00 \\
\hline
\end{tabular}

a Standard deviation

because of the numeric predominance of A-terminals in the gas phase. For $X_{\mathrm{g}}=0.25$ the probabilities of high $X_{\text {net }}$ is further increased (b), whereas for higher concentrations $\left(X_{\mathrm{g}}=0.9\right)$ the effect diminishes due to dilution (a). Statistical calculations giving the mean values and standard deviation of $X_{\text {net }}$ for the cases discussed above are shown in Table 2. The statistical nature of the results demonstrates that in most cases inclusion of $\mathrm{A}-\pi-\mathrm{A}$ guests is beneficial. It must be noted that for $\mathrm{D}-\pi-\mathrm{D}$ guests inclusion polarity is reversed but similar results are obtained.

\subsection{Beyond the idealized case}

Here we demonstrate the effects of (i) the host-guest interactions, (ii) the lateral guest-guest interactions, (iii) growth temperature and (iv) nonequal interactions for e.g. -A $\cdots$ D- between dipolar guests and $-\mathrm{A} \cdots \mathrm{D}-$ between dipolar and non-polar guests.

1. Host-guest interactions are accounted for by introducing an additional energy term, $\Delta E_{\mathrm{C}}$ in the exponent of the Boltzmann factors that determine the transition probabilities (Appendix 1). The host-guest interaction energy provides an alternative way by which polarity can arise in such systems. The parameter $\Delta E_{\mathrm{C}} \equiv E_{\mathrm{CA}}-E_{\mathrm{CD}}$ represents the energy difference experienced by the guest molecule depending on whether it enters the channel with the A- or D-terminal first. Its effect on polarity can be can be synergetic or antagonistic depending on whether it has the same or opposite sign with $\Delta E_{\mathrm{f}}$. Figure 6 illustrates the dependence of polarity on $\Delta E_{\mathrm{C}}$ in case all other interactions are set to zero.

2. In order to investigate the effect of lateral inter-channel guest-guest interactions, calculations considering a small perturbation were performed. For those calculations, the principal interaction energies were chosen randomly and the corresponding lateral guest-guest interaction energies, $\Delta E_{\perp} \equiv E_{\mathrm{p}}-E_{\mathrm{ap}}$, were chosen between 0 and $\pm 0.1, \pm 0.5$ and $\pm 1.0 \mathrm{~kJ} \mathrm{~mol}^{-1} . X_{\mathrm{g}}=0.5$ for $\mathrm{A}-\pi-\mathrm{A}$ and $T=300 \mathrm{~K}$. The results are shown in Fig. 7. The stronger the lateral interaction, the more the system deviates from the deterministic behaviour of Eq. 9 .

3. The attachment probabilities $P_{i j}$ resulting from inter-molecular interactions are essentially Boltzmann probability distributions, which are dependent on temperature. The system's microstates are not all equally probable [19]; thus the Boltzmann factor is biasing the distribution towards low energy states, i.e. as the temperature is reduced, the interaction probabilities are concentrated on a smaller group of low energy states. Therefore, temperature is a scaling factor to interaction energies 


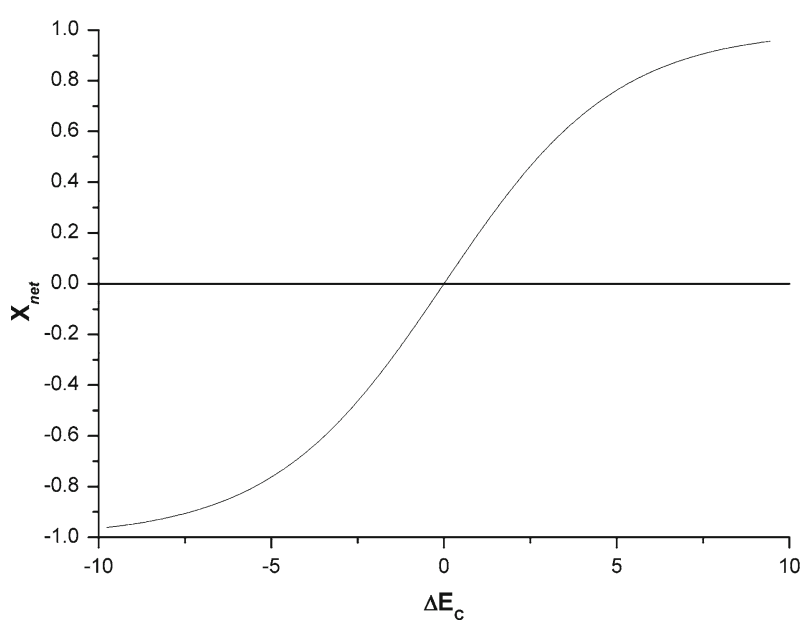

Fig. 6 The dependence of $X_{\text {net }}$ on $\Delta E_{\mathrm{C}}$, the guest to channel energy differences. All interaction energies are set to zero except for $E_{\mathrm{CA}}$ and $E_{\mathrm{CD}}$ which are randomly chosen within the range: $-5<E_{\mathrm{CA}}$, $E_{\mathrm{CD}}<5 \mathrm{~kJ} \mathrm{~mol}^{-1} . T=300 \mathrm{~K}$

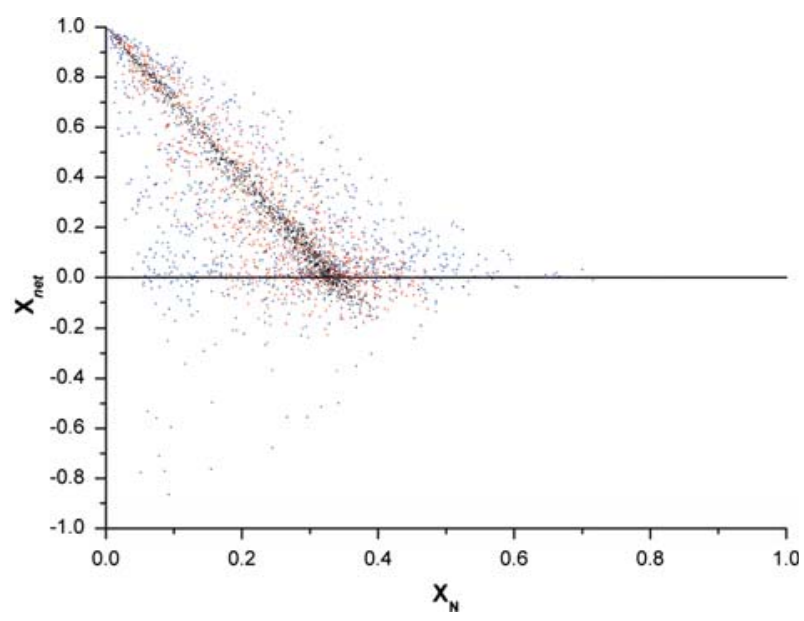

Fig. 7 The effect of lateral guest-guest interaction energies on polarity. Guest-guest interaction energies were randomly chosen in the range $-10<E_{i j}<2 \mathrm{~kJ} \mathrm{~mol}^{-1}$. The lateral interaction energies were selected from the range: $-0.1<\Delta E_{\perp}<0.1 \mathrm{~kJ} \mathrm{~mol}^{-1}$ (black points), $-0.5<\Delta E_{\perp}<0.5 \mathrm{~kJ} \mathrm{~mol}^{-1}$ (red points) and $-1.0<\Delta E_{\perp}<1.0 \mathrm{~kJ} \mathrm{~mol}^{-1}$ (blue points), $T=300 \mathrm{~K}$, here the $\mathrm{A}-\pi-\mathrm{A}$ guest concentration was $X_{\mathrm{g}}=0.5$. For colour, see electronic version

$E_{i j}$ and as such its variance cannot give rise to polarity. Instead, the variation of temperature influences the impact interaction energies and $X_{\mathrm{g}}$ have on polarity (see Fig. 8). We observe that depending on the initial concentration of non-polar guests, the effects of the growth temperature can be quite dramatic. The specific case of $X_{\mathrm{g}}=0.0003(\mathrm{D}-\pi-\mathrm{D})$ is rather representative: near room temperature no polarity arises. However, if the crystallisation would be carried out at lower or 


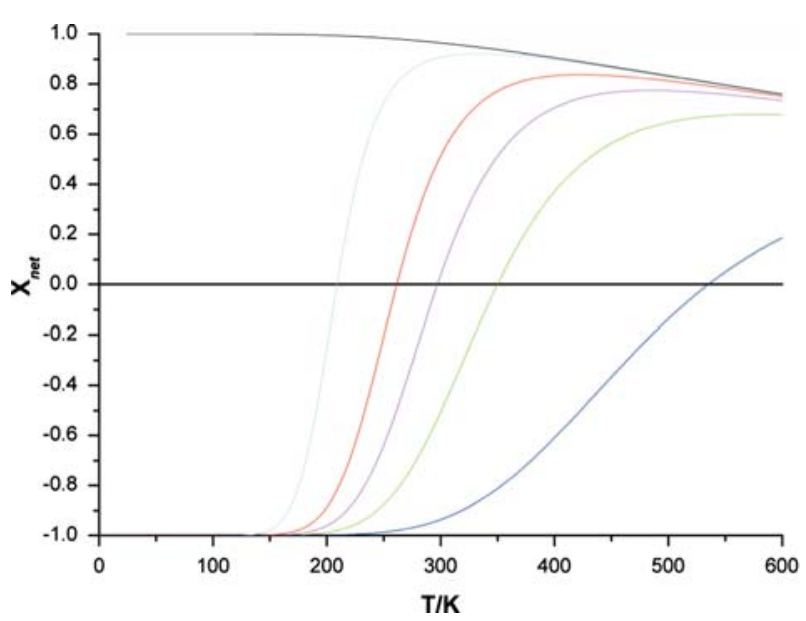

Fig. 8 The effect of temperature on polarity for various concentrations $X_{g}\left(-X_{\mathrm{g}}=0,-X_{\mathrm{g}}=0.00001\right.$, $\left.-X_{\mathrm{g}}=0.0001,-X_{\mathrm{g}}=0.0003,-X_{\mathrm{g}}=0.001,-X_{\mathrm{g}}=0.01\right)$. The interaction energies used for the calculations were $E_{\mathrm{AD}}=-25 \mathrm{~kJ} \mathrm{~mol}^{-1}, E_{\mathrm{AA}}=5 \mathrm{~kJ} \mathrm{~mol}^{-1}$ and $E_{\mathrm{DD}}=-5 \mathrm{~kJ} \mathrm{~mol}^{-1}$. For colour, see electronic version. Surprisingly, a very small amount of neutral $D-\pi-D$ molecules in the gas phase can have a strong effect on polarity as temperature is varied

higher temperatures, significant polarity could be achieved. The results of Fig. 8 were obtained assuming a Gibbs distribution and therefore only hold in thermal equilibrium, i.e. constant temperature of growth. If temperature is varied during growth the present description is invalid.

4. If we allow for non-equal interactions between dipolar guests and corresponding interactions between dipolar and non-polar guests thus removing the restrictions imposed on the selection of interaction energies, Eq. 6 no longer applies and the conclusions reached before are invalid. In such a system, the ratio of non-polar to dipolar guest molecules in the solid can be much higher than their ratio in the gas phase $(k>1)$. Furthermore, polarity is on average lower in such systems and more difficult to control.

\section{Conclusions}

The present study highlighted surprising aspects of polarity formation in channel-type hosts filled with dipolar and non-polar guest molecules. A universality behaviour is found i.e. macroscopic polarity can be determined by measuring the concentration of non-polar guests in the crystal without any further knowledge. It was also found that a very small concentration of non-polar guest molecules in the gas phase may have a dramatic impact on polarity. This brings up a delicate issue concerning the purity of $\mathrm{A}-\pi-\mathrm{D}$ used for inclusion formation. The results presented here suggest that particularly A- $\pi-\mathrm{A}$ impurities can be highly fortunate for polarity formation in channel-type inclusion compounds. Furthermore, the strength of this effect may be strongly temperature dependent. For $X_{\mathrm{g}}<0.01$ the theory predicts that the variation of the growth 
temperature can have a significant effect on macroscopic polarity. In extreme cases polarity may be reversed just by varying the growth temperature. Experimental work complementary to previously published $[11,16]$ findings is in progress.

Acknowledgement This work received partial support from NFP 47, Project No. 4047-057476/2.

\section{Appendix 1}

Substituting Eqs. 3 and 3 into $X_{\text {net }}=X_{\mathrm{A}}-X_{\mathrm{D}}$ yields:

$X_{\mathrm{net}}=\frac{P_{\mathrm{ND}}+P_{\mathrm{DD}} P_{\mathrm{NA}}-P_{\mathrm{DA}} P_{\mathrm{ND}}+P_{\mathrm{NA}} P_{\mathrm{AD}}-P_{\mathrm{NA}}-P_{\mathrm{ND}} P_{\mathrm{AA}}}{P_{\mathrm{ND}} P_{\mathrm{AA}}-P_{\mathrm{DD}} P_{\mathrm{AA}}+P_{\mathrm{DD}} P_{\mathrm{NA}}+P_{\mathrm{DA}} P_{\mathrm{AD}}-P_{\mathrm{DA}} P_{\mathrm{ND}}-P_{\mathrm{DA}}-P_{\mathrm{NA}} P_{\mathrm{AD}}+P_{\mathrm{NA}}-P_{\mathrm{AD}}+P_{\mathrm{ND}}+1}$

Substituting Eq. 3 for the probabilities in Eqs. 3 and A1 gives complicated expressions for $X_{\mathrm{N}}$ and $X_{\text {net }}$, respectively. Those quantities are now functions of the various interaction energies $E_{i j}$. In order to obtain the gradients of the linear curves of Fig. 3, one has to calculate the partial derivatives of $X_{\text {net }}$ and $X_{\mathrm{N}}$ with respect to the interaction energies involved in each expression:

$$
m=\frac{\partial X_{\text {net }} / \partial E_{i j}}{\partial X_{\mathrm{N}} / \partial E_{i j}}
$$

When considering the co-inclusion of A- $\pi-\mathrm{A}$ guests, Eq. A2 gives $m=\left(X_{\mathrm{g}}-2\right) / X_{\mathrm{g}}$ (or $m=-\left(X_{\mathrm{g}}-2\right) / X_{\mathrm{g}}$ in the case of $\mathrm{D}-\pi-\mathrm{D}$ guests) for all the derivatives irrespective of the interaction energies involved. Consequently, all points lay on a straight line as demonstrated in Fig. 3.

\section{References}

1. V. Ramamurthy, D.F. Eaton, Chem. Mater. 6, 1128-1136 (1994)

2. J. Hulliger, O. König, R. Hoss, Adv. Mater. 8, 719-721 (1995)

3. T. Müller, J. Hulliger, W. Seichter, E. Weber, T. Weber, M. Wübbenhorst, Chem. Eur. J. 6, 54-61 (2000)

4. T. Hertzsch, S. Kluge, E. Weber, F. Budde, J. Hulliger, Adv. Mater. 13, 1864-1867 (2001)

5. J. Hulliger, Z. Kristallogr. 214, 9-13 (1999)

6. A. Quintel, J. Hulliger, M. Wübbenhorst, J. Phys. Chem. B 102, 4277-4283 (1998)

7. P. Rechsteiner, J. Huliger, M. Flörsheimer, Chem. Mater. 12, 3296-3300 (2000)

8. H.B. Bürgi, J. Hauser, T. Weber, R.B. Neder, Cryst. Growth Des. 5, 2073-2083 (2005)

9. K.D.M. Harris, P. Jupp, Proc. R. Soc. Lond. A 453, 333-352 (1997); K.D.M. Harris, P. Jupp, Chem. Phys. Lett. 274, 525-534 (1998)

10. J. Hulliger, P. Rogin, A. Quintel, P. Rechsteiner, O. König, M. Wübbenhorst, Adv. Mater. 9, 677-680 (1997)

11. O. König, H. Bürgi, T. Armbruster, J. Hulliger, T. Weber, J. Am. Chem. Soc. 119, 10632-10640 (1997)

12. J.R. Norris, Markov Chains (Cambridge University Press, Cambridge, 1997)

13. T. Wüst, J. Hulliger, J. Chem. Phys. 122, 084715-1-084715-2 (2005)

14. C. Gervais, T. Hertzsch, J. Hulliger, J. Phys. Chem. B 109, 7961-7968 (2005)

15. J. Hulliger, S.W. Roth, A. Quintel, H. Bebie, J. Solid State Chem. 152, 49-56 (2000)

16. S.W. Roth, P.J. Langley, A. Quintel, M. Wübbenhorst, P. Rechsteiner, P. Rogin, O. König, J. Hulliger, Adv. Mater. 10, 1543-1546 (1998) 
17. T. Wüst, C. Gervais, J. Hulliger, Cryst. Growth. Des. 5, 93-97 (2005)

18. J. Hulliger, Chimia 55, 554-561 (2001)

19. J.P. Sethna, Statistical Mechanics: Entropy, Order Parameters, and Complexity (Oxford University Press, Oxford, 2006) 